\title{
Cascaded FRET in Conjugated Polymer/ Quantum Dot/Dye-Labeled DNA Complexes for DNA Hybridization Detection
}

\author{
Guoxin Jiang, Andrei S. Susha, ${ }^{\dagger}$ Andrey A. Lutich, Fernando D. Stefani, Jochen Feldmann, and \\ Andrey L. Rogach ${ }^{\text {t** }}$ \\ Photonics and Optoelectronics Group, Ludwig-Maximilians-Universität München, Amalienstrasse 54, 80799 Munich, Germany. ${ }^{\dagger}$ Present address: Department of Physics \\ and Materials Science, City University of Hong Kong, Tat Chee Avenue, Kowloon, Hong Kong
}

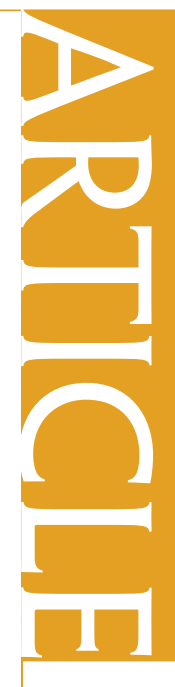

A $s$ the human genome project has uncovered the full sequence of human genomes and the postgenome technologies have been rapidly developed, DNA hybridization detection has become increasingly important. ${ }^{1}$ A variety of DNA detection formats are now available, including both heterogeneous assays with surface-bound array-based probes and homogeneous solution-based detection schemes. ${ }^{2}$ A number of those rely on fluorescence signal and, in particular, on fluorescence (or Förster) resonant energy transfer (FRET). ${ }^{3}$ Both conjugated polymers and semiconductor nanocrystals (colloidal quantum dots, QDs) have been suggested for use as fluorescent entities in DNA detection assays. ${ }^{4-12}$ Assays based on watersoluble cationic conjugated polymers allow one to utilize a simple binding strategy to DNA through electrostatic interactions with ionic side groups and benefit from the lightharvesting properties along the polymer backbone. ${ }^{4-8}$ QDs have been increasingly used as donors in FRET-related studies, including optical DNA diagnostics. ${ }^{9-12}$

QDs are photostable, highly efficient fluorophores with a strong band gap luminescence tunable by size as a result of the quantum confinement effect. ${ }^{13}$ FRET in QD/ DNA/organic dye conjugates has been used to study DNA hybridization, ${ }^{14,15}$ cleavage, ${ }^{16}$ and replication. ${ }^{17}$ Most of the reported approaches required covalent conjugation of DNA to the QD surface (e.g., by EDC/NHS coupling reaction), which often decreases the colloidal stability and/or the emission intensity of QDs. ${ }^{18,19}$ This limitation can be overcome by the use of a sensing platform www.acsnano.org
ABSTRACT Electrostatic complexes of a water-soluble fluorescent conjugated polymer, poly[9,9-bis $\left(3^{\prime}-((\mathrm{N}, \mathrm{N}-\right.$ dimethyl)-N-ethylammonium)propyl)-2,7-fluorene-alt-1,4-phenylene]dibromide (PDFD), and water-soluble CdTe quantum dots (QDs) are designed to provide a cascaded FRET for DNA hybridization detection. PDFD has two functions in the detection scheme: as a light-harvesting antenna, it enhances the emission of QDs by the first level FRET and inverts the sign of the surface charge of QDs, thus providing a positively charged surface to allow negatively charged dye-labeled DNA to interact with the resulting complex. This interaction causes the second level FRET to infrared-emitting dye labeled on the probe DNA, providing a reliable signal-on sensing platform discriminating between complementary and non-complementary DNA. A detailed spectroscopic study offers a clear description of photophysical processes in the designed polymer/QD/DNA complex, providing ample potential for further sensitivity and selectivity improvements.

KEYWORDS: homogeneous assay · DNA hybridization · semiconductor nanocrystals · conjugated polymers · FRET

relying on the electrostatic interaction of dye-labeled DNA with the oppositely charged QD surface ${ }^{20}$ in a similar fashion as in conducting polymer-based detection assays. $^{4-8}$

In this paper, we propose a simultaneous use of conjugated polymers and semiconductor QDs for DNA hybridization detection, which can potentially combine advantages of both light-harvesting and DNA-binding properties of water-soluble polymers with photostability, light-

harvesting ability, and FRET donor property of QDs. A blue-emitting conjugated polymer with positively charged side chains electrostatically self-assembles on the negatively charged surface of red-emitting CdTe QDs. The so-formed hybrid complex has a net positive charge and thus attracts negatively charged dye-labeled DNA molecules (Scheme 1). The water-soluble, cationic, conjugated polymer, poly[9,9-bis $\left(3^{\prime}-((N, N-\right.$
*Address correspondence to
andrey.rogach@cityu.edu.hk.

Received for review September 29, 2009 and accepted November 16, 2009.

Published online November 24, 2009. $10.1021 / \mathrm{nn} 901324 \mathrm{y}$

() 2009 American Chemical Society 


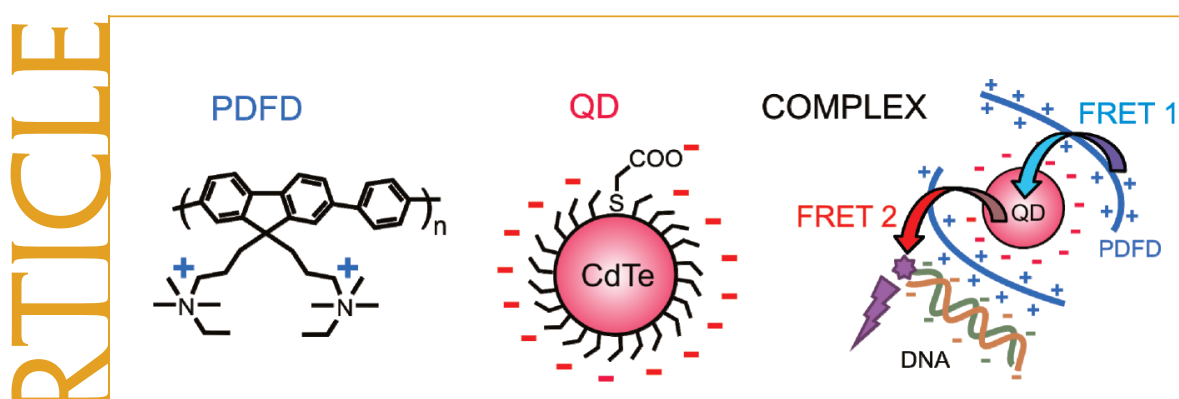

Scheme 1. Chemical structure of PDFD, schematics of a TGA-capped CdTe QD and of the PDFD/QD/dye-labeled DNA complex used to detect DNA hybridization through a cascaded double FRET. Upon optical excitation of PDFD and QDs, energy transfer takes place from the PDFD to the QDs (FRET 1) and from the QDs to the dye-labeled DNA (FRET 2). scription of photophysical processes in the designed polymer/QD/DNA complex.

\section{RESULTS AND DISCUSSION}

Upon titration of PDFD solution with CdTe QDs, emission of PDFD is quenched with a Stern-Volmer quenching constant of $1.2 \times$ $10^{8} \mathrm{M}^{-1}$ and almost disappears at the ratio of five PDFD molecules per QD. The

Stern-Volmer constant provides a measure for PL quenching efficiency and points out the formation of the PDFD/QD complex. ${ }^{6}$ At the same time, the QD photoluminescence (PL) is dimethyl)-N-ethylammonium)propyl)-2,7-fluorene-alt1,4-phenylene]dibromide (PDFD), is synthesized by a previously reported procedure with slight modifications. ${ }^{21}$ CdTe QDs (3.2 nm diameter) are synthesized directly in water as previously reported using thioglycolic acid (TGA) molecules as surface ligands (Scheme 1).22 TGA-capped CdTe QDs are strongly emitting room temperature quantum yield of the sample used here is $43 \%$ ), stable in water and common buffers, ${ }^{23}$ as the complexes reported here are, and negatively charged at slightly basic $\mathrm{pH} .{ }^{24}$ Fluorescent labeled DNA is used to monitor the interaction of the PDFD/QD complex and DNA through a double FRET process (Scheme 1): FRET 1 from PDFD to QDs and FRET 2 from QDs to the dye on the DNA. The infrared dye IRD700 is selected as the acceptor dye because its absorbance has no spectral overlap with the emission of PDFD but a sufficient overlap with the emission of QDs (Figure 1a). A detailed spectroscopic study reported here provides a clear de-
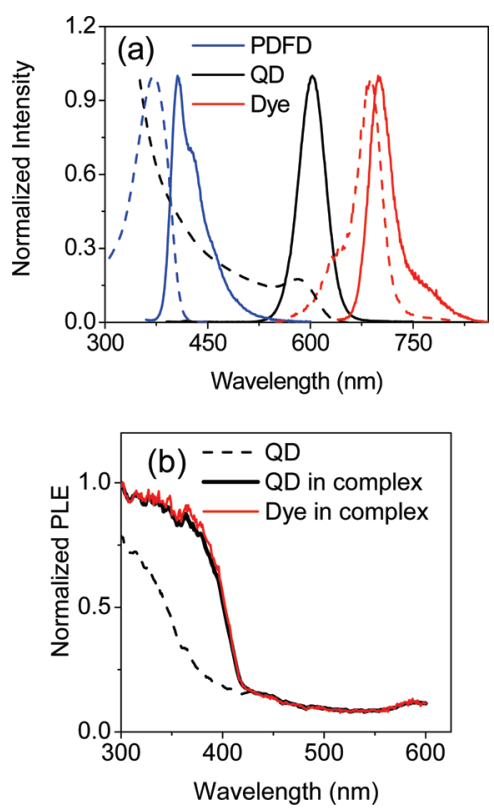

Figure 1. (a) Normalized absorption (dashed lines) and emission (solid lines) spectra of PDFD, CdTe QDs, and IRD700 dye. (b) Normalized PL excitation spectra detected at the emission wavelength of bare QDs, QDs in PDFD/QD complex, and IRD700-labeled ds-DNA in PDFD/QD/DNA complex. enhanced due to the FRET 1. The binding of PDFD and QDs is confirmed by the inversion of electrophoretic mobility of the colloidal solution from $-3.89 \times 10^{-8}$ to $+1.57 \times 10^{-8} \mathrm{~m}^{2} / \mathrm{Vs}$ upon titration of PDFD with QDs. The PDFD and QD concentrations are fixed at $2.5 \times$ $10^{-7}$ and $5 \times 10^{-8} \mathrm{M}$, respectively, for further measurements.

The occurrence of energy transfer between the PDFD and QDs in the PDFD/QD complex (FRET 1) as well as between the PDFD/QD complex and IRD700 dye (FRET 2) is confirmed by PL excitation (PLE) spectroscopy (Figure 1b). First, by comparing the behavior of bare QDs and QDs in the PDFD/QD complex, it can be clearly seen how the excitation of QDs increases when the absorption of PDFD sets at wavelengths below 425 $\mathrm{nm}$, demonstrating the presence of the FRET 1 process. Second, in the PDFD/QD/DNA complex, the shape of the PLE spectra of the IRD700-labeled double-stranded (ds) DNA perfectly matches the one of QDs, revealing the existence of the FRET 2 process. The energy transfer from the QDs to the dye-labeled DNA is evidenced by the faster decay of the QD emission as the concentration of dye-labeled DNA increases (Figure 2a). At the same time, the emission of the IRD700 dye becomes slower due to the delayed excitation provided by feeding from QDs through FRET 2 (Figure 2b).

In order to quantify efficiency of the FRET 1 from PDFD to QDs, steady-state absorption and PL measurements have been performed on bare QDs and on a PDFD/QD complex. Considering the PL increase of QDs upon addition of PDFD, the FRET 1 efficiency can be calculated using the following relation:

$$
\Phi_{\mathrm{FRET} 1}=\frac{A_{\mathrm{QD}}\left(\lambda_{\text {exc }}\right)}{A_{\mathrm{PDFD} / \mathrm{QD}}\left(\lambda_{\mathrm{exc}}\right)-A_{\mathrm{QD}}\left(\lambda_{\mathrm{exc}}\right)}\left(\frac{l_{\mathrm{QD}}}{l_{\mathrm{QD}}^{0}}-1\right)
$$

where $A_{\mathrm{QD}}\left(\lambda_{\text {exc }}\right)$ and $A_{\mathrm{PDFD} / \mathrm{QD}}\left(\lambda_{\text {exc }}\right)$ are absorption of the acceptor (bare QDs) and of the donor-acceptor pair (PDFP/QD composite), respectively, at the wavelength of excitation $\lambda_{\text {exc. }} l_{\mathrm{QD}}^{0}$ and $l_{\mathrm{QD}}$ are $\mathrm{PL}$ intensities of the QDs in absence and presence of the PDFD, respectively. Applying eq 1 to the absorption and PL data, $\Phi_{\text {FRET1 }}$ equals 72\%. Time-resolved PL measurements considered in de- 

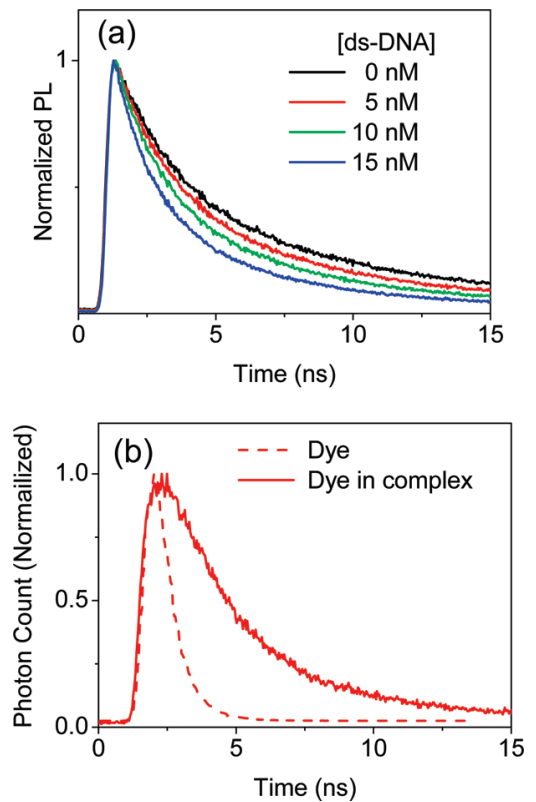

Figure 2. (a) Time-resolved PL decays of $Q D$ emission in the PDFD/QD complex (excited at $400 \mathrm{~nm}$ ) incubated with different concentrations of dye-labeled ds-DNA, as indicated in the frame. (b) Time-resolved PL decays of bare IRD700 dye excited directly at $680 \mathrm{~nm}$ and of IRD700-labeled ds-DNA (5 $\mathrm{nM}$ ) in PDFD/QD/DNA complex, excited at $400 \mathrm{~nm}$.

tail in ref 25 have confirmed that the FRET 1 from the PDFD to the QDs is a very fast ( $\sim 50 \mathrm{ps}$ ) process.

The efficiency of the FRET 2 from QDs to infrared dye was estimated by monitoring the reduction of the PL intensity of QDs caused by energy transfer to dye molecules using the following relation:

$$
\Phi_{\mathrm{FRET2}}=1-\frac{I_{\mathrm{QD}}}{I_{\mathrm{QD}}^{0}}
$$

where $I_{\mathrm{QD}}^{0}$ and $I_{\mathrm{QD}}$ are the PL intensities of QDs in absence and presence of $5 \mathrm{nM}$ of a complementary dsDNA, respectively. Applying eq 2 to the measured absorption and PL spectra, we found $\Phi_{\text {FRET2 }}$ to be $42 \%$. Assuming random orientations of dipole moments of donors and acceptors, we estimated a Förster radius of $5.8 \mathrm{~nm}$ for the PDFD/QD pair and $5.1 \mathrm{~nm}$ for the QD/dye pair.

To demonstrate the ability of PDFD/QD complex for DNA hybridization detection, the former is titrated with (i) probe single-stranded (ss) DNA labeled with IRD700 dye, (ii) probe DNA incubated with a noncomplementary DNA, and (iii) probe DNA incubated with a complementary DNA to produce ds-DNA. Figure 3a shows PL spectra of the PDFD/QD complex upon incubation with an increasing amount of IRD700labeled ds-DNA, which displays a progressive loss in QD emission alongside with an increase of the emission intensity of IRD700 dye, being consistent with FRET 2 from PDFD/QD complex to the dye. The ratio of the emission intensities of IRD700 dye and QD (Figure 3b) is proportional to the concentration of DNA, thus allowwww.acsnano.org
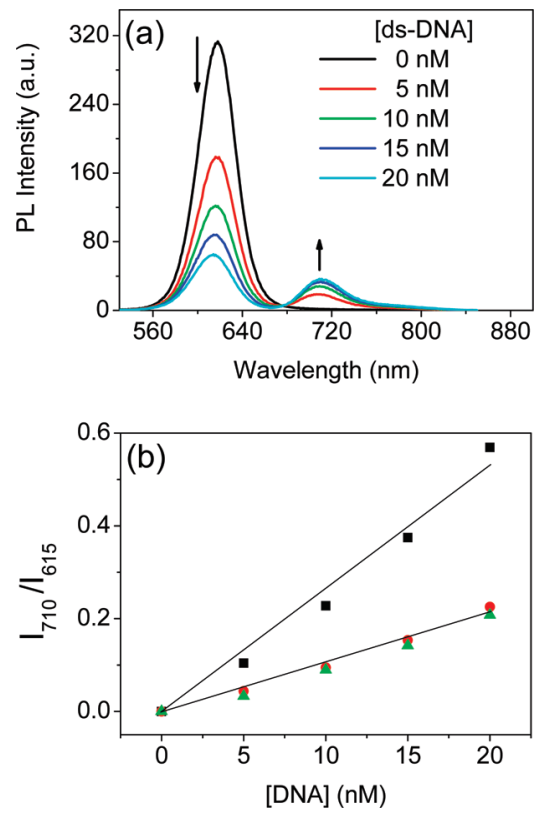

Figure 3. (a) Emission spectra of the PDFD/QD/DNA complex for different concentrations of dye-labeled complementary ds-DNA, as indicated in the frame. (b) Ratio of the emission intensity of IRD700 dye (peak at $710 \mathrm{~nm}$ ) and CdTe QDs (peak at $615 \mathrm{~nm}$ ) vs DNA concentration of ss-DNA (green triangles), non-complementary DNA (red circles), and complementary ds-DNA (black squares).

ing a quantitative assay. Importantly, the proportionality constant for complementary DNA is 2.5 times larger than for ss-DNA, allowing us to unambiguously detect the DNA hybridization event. This is a consequence of the higher local charge density of ds-DNA that leads to a stronger electrostatic binding to the PDFD/QD complex. The detection limit of our system as demonstrated here ( $<5 \mathrm{nM}$ ) favorably compares with previously reported values for QD-based DNA hybridization assays. ${ }^{15,26}$ The dual use of both conjugated polymer and QD extinction offers a potential to lower this limit to picomolar range. ${ }^{4,5,8}$

\section{CONCLUSION}

In summary, a facile approach has been demonstrated for the detection of DNA hybridization, which is based on a cascaded double FRET from a complex of conjugated polymer and CdTe QDs to the dye-labeled DNA. The conjugated polymer provides a dual advantage of (i) a light-harvesting antenna enhancing the emission of QDs and thus potentially offering improved assay sensitivity and (ii) inverting the sign of originally negatively charged QDs and thus providing a positively charged counterpart for negatively charged DNA molecules to electrostatically adsorb on polymer/QD complexes. The difference in electrostatic interaction strength for singlestranded and double-stranded DNA results in different slopes of a linearly increasing ratio of dyeto-QD emission intensities, providing a reliable signal-on sensing platform for the detection of hy- 
bridization events. Our detailed spectroscopic analysis offers a clear description of FRET processes taking place in the polymer/QD/DNA complex and offers ample potential for future sensitivity improvements of the detection platform proposed. It can also be useful for the development of an alternative strategy with an improved selectivity of DNA detection, with cascaded FRET processes taking place to intercalated dyes, thus amplifying optical response from ethidium bromide (EB)-based DNA protocols. ${ }^{27}$

\section{EXPERIMENTS}

Poly[9,9-bis ( $3^{\prime}$-((N,N-dimethyl)- $N$-ethylammonium)propyl)2,7-fluorene-alt-1,4-phenylene]dibromide (PDFD) was synthesized by following a previously reported procedure ${ }^{21}$ with a slight modification. The bromo monomer, 2,7-dibromo-9,9bis( $3^{\prime}$-( $N, N$-dimethylamino) propyl)fluorene, was purified by silica gel chromatography instead of recrystallization. The quaternization of the neutral precursor of the polymer was carried out in a mixture of THF and DMSO ( $/ \mathrm{v}=3: 1)$. The solution was stirred at $50^{\circ} \mathrm{C}$ for 5 days. During the reaction period, intermittent addition of a certain amount of water was needed to dissolve any precipitation seen in the solution in order to achieve a high yield of quaternization. After the reaction was finished, THF, water, and extra bromoethane were evaporated in vacuum. The remaining DMSO solution containing the target polymer was precipitated by pouring into a large amount of diethyl ether. The precipitate was collected by centrifugation, washed with acetone, and dried overnight at $80^{\circ} \mathrm{C}$ in a vacuum oven. The assynthesized water-soluble polymer PDFD inherits the advantages of excellent chemical stability and high fluorescence of classic oil-soluble polyfluorene. ${ }^{28}$ The structure of the PDFD was corroborated by ${ }^{1} \mathrm{H} \mathrm{NMR}(300 \mathrm{MHz}$, DMSO- $d)$ : $\delta 7.8-7.4(\mathrm{~m}, 10 \mathrm{H})$, $3.15-3.10(\mathrm{~m}, 8 \mathrm{H}), 2.87-2.77(\mathrm{~m}, 16 \mathrm{H}), 2.63(\mathrm{~m}, 4 \mathrm{H}), 0.98(\mathrm{~m}$, $10 \mathrm{H}$ ). The molecular weight of the neutral precursor of PDFD was determined to be $M_{\mathrm{w}}=19.0 \mathrm{kDa}(\mathrm{PDI}=1.9)$ by GPC using polystyrene as standard reference and THF as eluent.

Electrophoretic mobility of QDs and PDFD/QD complex has been determined by a ZetaSizer (Malvern Instruments). Absorption spectra were recorded with a Cary 5000 UV-vis-NIR spectrometer (Varian). Steady-state fluorescence spectra were measured with a Cary Eclipse fluorescence spectrometer (Varian). Time-resolved PL spectra of QDs and dyes were measured using time-correlated singlephoton counting (Time-Harp, PicoQuant). The samples were excited by picosecond pulses generated by a PicoQuant, LDH-400 laser head controlled by a PDL-800B driver. The setup was operated with an overall time resolution of $150 \mathrm{ps}$. PL decays were measured to $5000-6000$ counts in the peak. Timeresolved PL spectra of the polymer PDFD were measured with a streak camera (Hamamatsu C5680) combined with the spectrometer (Cromex with $40 \mathrm{gr} / \mathrm{mm}$ grating). The Hamamatsu Syncroscan Sweep Unit M5675 and Slow Speed Sweep Unit M5677 were used to detect PL kinetics in different temporal window from 0.5 to $100 \mathrm{~ns}$, respectively. The frequency-doubled output of the mode-locked titanium:sapphire laser ( $120 \mathrm{fs}, 76.5 \mathrm{MHz}$ ) was used as an excitation source in the range from 370 to $470 \mathrm{~nm}$.

The DNA was purchased from Metabion GmbH, Germany, and the sequences used in this work were as follows: (i) probe DNA: IRD700 5'-GAT GAG TAT TGA TGC CGA-3'; (ii) complementary DNA: 5'-TCG GCA TCA ATA CTC ATC-3'; (iii) noncomplementary DNA: 5'-TAT GCT GGT GCG TCG CAC-3'. Equal amounts of probe DNA (ss-DNA) and complementary or noncomplementary DNA were mixed together and incubated at 38 ${ }^{\circ} \mathrm{C}$ for $45 \mathrm{~min}$. The resulting solutions $\left(1 \times 10^{-6} \mathrm{M}\right)$ were subsequently titrated into the PDFD/QD complex prepared by mixing of $10 \mu \mathrm{L}$ of $10^{-5} \mathrm{M}$ QDs and $2 \mathrm{~mL}$ of $2.5 \times 10^{-7} \mathrm{M}$ PDFD.

Acknowledgment. We acknowledge financial support of the DFG via the "Nanosystems Initiative Munich" (NIM), the LMUexcellent program, and the project RO2345/5-1. G.J. and A.A.L. thank Alexander von Humboldt Foundation for a research fellowship.

\section{REFERENCES AND NOTES}

1. Hood, L.; Galas, D. The Digital Code of DNA. Nature 2003, 421, 444-448.

2. Epstein, J. R.; Biran, I.; Walt, D. R. Fluorescence-Based Nucleic Acid Detection and Microarrays. Anal. Chim. Acta 2002, 469, 3-36.

3. Lakowicz, J. R. Principles of Fluorescence Spectroscopy; Kluwer Academic/Plenum Publishers: New York, 1999.

4. Gaylord, B. S.; Heeger, A. J.; Bazan, G. C. DNA Detection Using Water-Soluble Conjugated Polymers and Peptide Nucleic Acid Probes. Proc. Natl. Acad. Sci. U.S.A. 2002, 99, 10954-10957.

5. Chi, C.; Mikhailovsky, A.; Bazan, G. C. Design of Cationic Conjugated Polyelectrolytes for DNA Concentration Determination. J. Am. Chem. Soc. 2007, 129, 11134-11145.

6. Li, H.; Yang, R.; Bazan, G. C. Fluorescence Engergy Transfer to Dye-Labeled DNA from a Conjugated Polyelectrolyte Prequenched with a Water-Soluble C60 Derivative. Macromolecules 2008, 41, 1531-1536.

7. Nayak, R. R.; Nag, O. K.; Kang, M.; Jin, Y.; Suh, H.; Lee, K.; Woo, H. Y. A Micellar Complex of a Conjugated Polyelectrolyte for Efficient FRET to Dye-Labeled DNA. Macromol. Rapid Commun. 2009, 30, 633-638.

8. Zheng, W.; He, L. Label-Free, Real-Time Multiplexed DNA Detection Using Fluorescent Conjugated Polymers. J. Am. Chem. Soc. 2009, 131, 3432-3433.

9. Algar, W. R.; Massey, M.; Krull, U. J. The Application of Quantum Dots, Gold Nanoparticles and Molecular Switches to Optical Nucleic-Acid Diagnostics. Trends Anal. Chem. 2009, 28, 292-306.

10. Franzl, T.; Klar, T. A.; Schietinger, S.; Rogach, A. L.; Feldmann, J. Exciton Recycling in Graded Gap Nanocrystal Structures. Nano Lett. 2004, 4, 1599-1603.

11. Medintz, I. L.; Clapp, A. R.; Mattoussi, H.; Goldman, E. R.; Fisher, B.; Mauro, J. M. Self-Assembled Nanoscale Biosensors Based on Quantum Dot FRET Donors. Nat. Mater. 2003, 2, 630-638.

12. Rogach, A. L.; Klar, T. A.; Lupton, J. M.; Meijerink, A.; Feldmann, J. Energy Transfer with Semiconductor Nanocrystals. J. Mater. Chem. 2009, 19, 1208-1221.

13. Rogach, A. L., Ed. Semiconductor Nanocrystal Quantum Dots; Springer: New York, 2008.

14. Algar, W. R.; Krull, U. J. Interfacial Transduction of Nucleic Acid Hybridization Using Immobilized Quantum Dots as Donors in Fluorescence Resonance Energy Transfer. Langmuir 2009, 25, 633-638.

15. Wu, C.-S.; Cupps, J. M.; Fan, X. Compact Quantum Dot Probes for Rapid and Sensitive DNA Detection Using Highly Efficient Fluorescence Resonant Energy Transfer. Nanotechnology 2009, 20, 305502-1-305502-7.

16. Gill, R.; Willner, I.; Shweky, I.; Banin, U. Fluorescence Resonance Energy Transfer in CdSe/ZnS-DNA Conjugates: Probing Hybridization and DNA Cleavage. J. Phys. Chem. B 2005, 109, 23715-23719.

17. Patolsky, F.; Gill, R.; Weizmann, Y.; Mokari, T.; Banin, U.; Willner, I. Lighting-Up the Dynamics of Telomerization and DNA Replication by CdSe-ZnS Quantum Dots. J. Am. Chem. Soc. 2003, 125, 13918-13919.

18. Algar, W. R.; Krull, U. J. Towards Multi-Colour Strategies for the Detection of Oligonucleotide Hybridization Using Quantum Dots as Energy Donors in Fluorescence Resonance Energy Transfer (FRET). Anal. Chim. Acta 2007, 581, 193-201. 
19. Zhou, D. J.; Ying, L. M.; Hong, X.; Hall, E. A.; Abell, C.; Klenerman, D. A Compact Functional Quantum Dot-DNA Conjugate: Preparation, Hybridization, and Specific Label-Free DNA Detection. Langmuir 2008, 24, 1659-1664.

20. Lee, J.; Choi, Y.; Kim, J.; Park, E.; Song, R. Positively Charged Compact Quantum Dot-DNA Complexes for Detection of Nucleic Acids. ChemPhysChem 2009, 10, 806-811.

21. Huang, F.; Wu, H. B.; Wang, D.; Yang, W.; Cao, Y. Novel Electroluminescent Conjugated Polyelectrolytes Based on Polyfluorene. Chem. Mater. 2004, 16, 708-716.

22. Rogach, A. L.; Franzl, T.; Klar, T. A.; Feldmann, J.; Gaponik, N.; Lesnyak, V.; Shavel, A.; Eychmuller, A.; Rakovich, Y. P.; Donegan, J. F. Aqueous Synthesis of Thiol-Capped CdTe Nanocrystals: State-of-the-Art. J. Phys. Chem. C 2007, 111, 14628-14637.

23. Gaponik, N.; Radtchenko, I. L.; Gerstenberger, M. R.; Fedutik, Y. A.; Sukhorukov, G. B.; Rogach, A. L. Labeling of Biocompatible Polymer Microcapsules with Near-Infrared Emitting Nanocrystals. Nano Lett. 2003, 3, 369-372.

24. Nabiev, I.; Mitchell, S.; Davies, A.; Williams, Y.; Kelleher, D.; Moore, R.; Gun'ko, Y. K.; Byrne, S.; Rakovich, Y. P.; Donegan J. F.; et al. Nonfunctionalized Nanocrystals Can Exploit a Cell's Active Transport Machinery Delivering Them to Specific Nuclear and Cytoplasmic Compartments. Nano Lett. 2007, 7, 3452-3461.

25. Lutich, A. A.; Jiang, G.; Stefani, F. D.; Susha, A. S.; Rogach, A. L.; Feldmann, J. Energy Transfer versus Charge Separation in Type-II Hybrid Organic-Inorganic Nanocomposites. Nano Lett. 2009, 9, 2636-2640.

26. Peng, H.; Zhang, L. J.; Kjallman, T. H. M.; Soeller, C.; TravasSejdic, J. DNA Hybridization Detection with Blue Luminescent Quantum Dots and Dye-Labeled SingleStranded DNA. J. Am. Chem. Soc. 2007, 129, 3048-3049.

27. Liu, B.; Dan, T. T. T.; Bazan, G. C. Collective Response from a Cationic Tetrahedral Fluorene for Label-Free DNA Detection. Adv. Funct. Mater. 2007, 17, 2432-2438.

28. Neher, D. Polyfluorene Homopolymers: Conjugated Liquid-Crystalline Polymers for Bright Blue Emission and Polarized Electroluminescence. Macromol. Rapid Commun. 2001, 22, 1365-1385. 